Research Paper

\title{
Loss of LIMCHI predicts poor prognosis in patients with surgically resected Lung Adenocarcinoma: A study based on Immunohistochemical Analysis and Bioinformatics
}

\author{
He Cao ${ }^{1}$, Jing Zhao ${ }^{2}$, Zhen Chen², Wenjia Sun ${ }^{1}$, Kexin Ruan ${ }^{1}$, Jianya Zhou ${ }^{1 凶}$ and Jianying Zhou ${ }^{1 凶}$ \\ 1. Department of Respiratory Disease, Thoracic Disease Center, The First Affiliated Hospital, College of Medicine, Zhejiang University, Hangzhou 310003, \\ China. \\ 2. Department of Pathology, The First Affiliated Hospital, College of Medicine, Zhejiang University, Hangzhou 310003, China.
}

$\square$ Corresponding authors: Jianya Zhou (E-mail: zhoujy@zju.edu.cn) or Jianying Zhou (E-mail: zjyhz@zju.edu.cn), Department of Respiratory Disease, Thoracic Disease Center, The First Affiliated Hospital, College of Medicine, Zhejiang University, No.79, Qingchun Road, Shangcheng District, Hangzhou, Zhejiang 310003, China. Tel: +86-0571-87236876; Fax: +86-0571-87236876.

(1) The author(s). This is an open access article distributed under the terms of the Creative Commons Attribution License (https://creativecommons.org/licenses/by/4.0/). See http:/ /ivyspring.com/terms for full terms and conditions.

Received: 2020.05.07; Accepted: 2020.10.13; Published: 2021.01.01

\begin{abstract}
Background: LIMCHI, a novel actin-binding protein, is reported to correlate with tumorigenesis in multiple cancer types, but its clinical prognostic value in lung adenocarcinoma (LUAD) patients remains unclear.

Methods: A total of 196 patients with LUAD who underwent R0 resection were included for analysis. We integrated immunohistochemistry (IHC) and data mining analyses to determine LIMCHI expression in tumor specimens; the chi-square test was used to explore the correlation between clinicopathologic factors and LIMCHI expression in LUAD; Kaplan-Meier curves and the Cox proportional hazards model were used to investigate the clinical prognostic role of LIMCHI expression in patients with LUAD; and DAVID enrichment and gene set enrichment analysis (GSEA) were used to determine the underlying molecular mechanism.

Results: LIMCHI protein and mRNA expressions were significantly decreased in LUAD tissues. LIMCHI mRNA expression was a potential diagnostic indicator in the TCGA cohort, and was associated with poor prognosis. IHC results in our LUAD cohort demonstrated that the LIMCHI expression level was significantly associated with pleural invasion, tumor length, tumor differentiation grade, and clinical tumor stage. Patients with higher LIMCHI expression had longer overall survival times. Cox multivariate survival analysis showed that LIMCHI expression independently predicted the outcome. GO and KEGG clustering analyses showed that LIMCHI-related genes may be involved in 'cell adhesion', 'signal transduction', and several cancer-related pathways. GSEA showed 8 enriched hallmarks in the low LIMCHI expression group, including mTOR signaling, MYC signaling, DNA repair, and G2M checkpoint.

Conclusions: Our findings suggest that LIMCHI may serve as a promising biomarker to predict LUAD prognosis.
\end{abstract}

Key words: lung adenocarcinoma, LIMCH1, TCGA, immunochemistry, prognosis

\section{Introduction}

Lung cancer is one of the most common cancer types, and is a major threat to global health. Lung adenocarcinoma (LUAD) and lung squamous carcinoma (LUSC), the main histological forms of lung cancer, rank as the most aggressive malignancies. Patients with LUAD account for $\sim 50 \%$ of all lung cancer cases, and the rate is continually increasing $[1,2]$. Based on cancer statistics, 1.8 million 
deaths worldwide are attributed to lung cancer, and LUAD accounts for $\sim 40 \%$ of these cases [3]. Rapid progress in molecular genetics has begun to reveal genetic mechanisms involved in lung cancer. Genetic factors have been shown to play a pivotal role in LUAD tumorigenesis. As a result of breakthroughs in molecular genetics, EGFR-tyrosine kinase inhibitors (TKIs) and PD-1/PD-L1 inhibitors have made significant breakthroughs in treating patients with LUAD. However, not all patients with LUAD are able to benefit from these treatments, and the survival rate is still unsatisfactory. Other reliable biomarkers are urgently needed.

Actin-binding proteins regulate various aspects of actin dynamics, and many actin dynamic- related genes have been reported to play critical roles in the development of tumors. Espin (ESPN), an actinfilament-binding protein, regulates organization, actin dynamics, and signaling transduction in the actin filament-rich cells. $\mathrm{Li}$ et al. reported that decreases in endogenous ESPN slowed esophageal squamous cell carcinoma (ESCC) cell growth and suggested ESPN as a novel therapeutic target [4]. Twinfilin-1 (TWF1) is another conserved actinbinding protein, which is overexpressed in non-smallcell lung cancer (NSCLC), and is an independent predictor of poor outcomes in patients with LUAD [5].

LIMCH1 is located on chromosome 4p13 and encodes a novel actin-binding protein. It is expressed broadly in many human organs, including the lungs, spleen, heart, and brain. LIMCH1 could directly bind to non-muscle myosin II (NM-II) to regulate the NM-II activity, which is involved in cell movement. The depletion of LIMCH1 in HeLa cells attenuated the number of actin stress fibers and increased cell migration ability [6]. Recent studies have reported its specific tumorigenesis role in several cancer types. Bersini et al. identified that low expression of LIMCH1 were associated with advanced tumor stages in patients with triple-negative breast cancer [7]. In clear cell renal cell carcinoma (ccRCC), Eckel-Passow and colleagues found that LIMCH1 expression can increase the risk of smoking-related ccRCC [8]. As for lung cancer, Liu et al. performed analyses in silicon and found downregulation of LIMCH1 mRNA expression in LUAD tissues compared with normal tissues [9]. In another study, Zhang et al. confirmed that LIMCH1 mRNA expression was decreased in clinical NSCLC samples. Overexpression of LIMCH1 inhibited the growth of the A549 cell line [10]. A previous study conducted by Karlsson et al. revealed a new biomarker of the paralogous protein LMO7 [11]. Nonetheless, the clinical prognostic value of LIMCH1 in patients with LUAD remains unclear.

Therefore, in this study, we used immunohistochemistry (IHC) to examine the prognostic relevance of LIMCH1 expression in our large LUAD cohort. We also explored the underlying molecular mechanisms by bioinformatics analysis.

\section{Methods}

\section{Patients and Tissue Samples}

We retrospectively collected clinical data and tissue samples for 196 patients with LUAD who underwent R0 resection between January 2011 and March 2013 at the First Affiliated Hospital of Zhejiang University in Hangzhou, China. We also collected 9 matched adjacent normal tissues. We excluded patients who were lost to regular follow-up, had incomplete clinical information, or had ever received chemotherapy or radiotherapy before surgery (Figure 1). All clinical data in this study were collected from the hospital's electronic medical record system. The hospital's ethics committee approved the study, and informed consents had previously been provided before the index surgery.

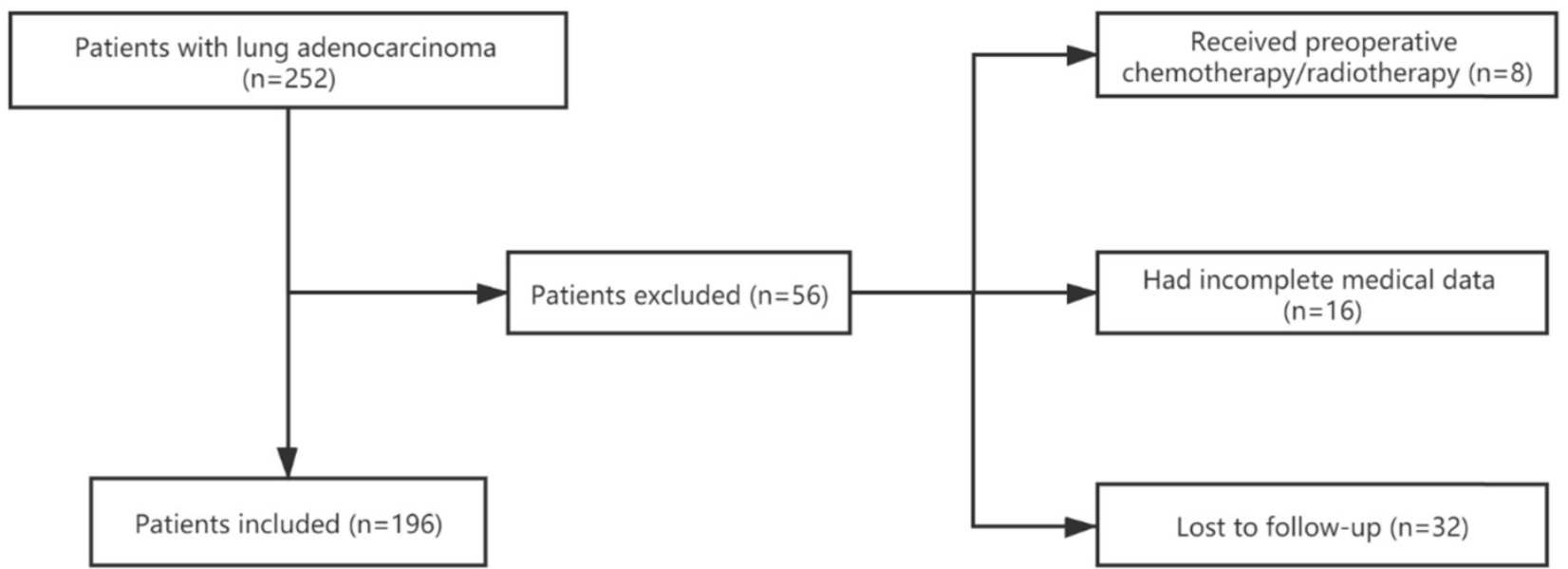

Figure 1. Flowchart of the enrollment process. 
All of the samples selected were pathologically confirmed as LUAD. Histological tumor grading and classifications were evaluated according to the World Health Organization's (WHO) grading system [12]. Tumor data included clinical stage, location, length, and differentiation. Patient data included sex, age, smoking history, and length of survival. The last follow-up was in December 2019.

\section{Tissue Microarray and IHC Staining}

A 3-mm-diameter core of a representative tumor area was selected to construct the tissue microarray (TMA). For IHC staining, TMA slides were de-paraffinized with xylene and then rehydrated with graded ethanol, after which antigens were retrieved. Endogenous peroxidase activity was reduced by saturating it with 3\% hydrogen peroxide, and the slides were blocked in goat serum. After blocking, the slides were stained with the pre-diluted anti-LIMCH1 antibody (Abcam, ab96178). The next day, 3,3diaminobenzidine (DAB) was used according to the manufacturer's instructions to stain the samples.

IHC results were inspected by 2 pathologists, who were blinded to the patient data. The LIMCH1 staining scores were semi-quantified using a modified immunoreactive scoring (IRS) system based on the staining intensity and percentage of epithelial cells within the tumor [13]. Intensity was detected as no staining (0), weak (1), moderate (2), or strong (3). Immunoreactivity was detected as no staining (0), $1 \%-25 \%$ (1), $26 \%-50 \%$ (2), $51 \%-75 \%$ (3), or $>75 \%$ (4); (see Figure 3E-L). The final IHC score was then calculated by multiplying the grades for the low (score $<7$ ) and high (score $\geq 7$ ) groups.

\section{LIMCHI Expression in Public Datasets}

We downloaded the expression profile and clinical information of the GDC TCGA-LUAD cohort from UCSC Xena (https://xena.ucsc.edu/) [14]. Data from 524 primary tumors in patients with LUAD and 59 controls were obtained for RNA-seq analysis. Of these, who had a primary tumor, 511 cases had intact overall survival (OS) data. We extracted the demographic and clinical parameters for these patients. We also screened the expression of LIMCH1 between LUAD and normal groups by using Oncomine (https://www.oncomine.org/) [15]. This online dataset provides access to the molecular profiling data for tissues and cells from many microarray chips. We also downloaded the LIMCH1 protein expression from the Human Protein Atlas (http://www.proteinatlas.org) [16, 17].

\section{Clustering Analysis of LIMCHI Co-expressed Genes}

To investigate the potential mechanisms responsible for LIMCH1 expression, we obtained LIMCH1-related co-expressed genes from the MEM (http://biit.cs.ut.ee/mem/index.cgi) [18] and cBioPortal databases (http://www.cbioportal.org) [19]. We extracted the results from the top 5000 coexpressed genes in MEM. In cBioPortal, 20034 genes were co-expressed with LIMCH1 in LUAD (TCGA, Provisional). Of these, 1303 genes were included after being filtered with an absolute value of the Pearson's correlation coefficient $>0.3$. The intersection of the results from MEM and cBioPortal was then visualized using the Venny tool (http://bioinfogp.cnb.csic.es/ tools/venny/index.html) [20]. Next, the geneannotation enrichment analysis and functional annotation clustering of these co-expressed genes were analyzed using the DAVID Bioinformatics Resources 6.8 database (https://david.ncifcrf.gov/) [21].

\section{Gene Set Enrichment Analysis}

Gene Set Enrichment Analysis (GSEA) was performed by using GSEA v3.0 software from the Broad Institute, which computes 1000 permutations to find whether significant and concordant differences exist between 2 physiological states. Patients with LUAD were grouped by the median expression of LIMCH1, and h.all.v7.2.symbols.gmt was used as the reference gene set. A gene set is considered to be significantly enriched when a normal $P$ value is $<0.05$, and a false discovery rate (FDR) is $<0.25$.

\section{Statistical Analysis}

The data were analyzed using SPSS 22.0. The clinicopathological data were compared using the chi-squared test or the two-sided Student's t-test. Receiver operating characteristic (ROC) curves were used to assess diagnostic ability, the Kaplan-Meier method was used for overall survival analysis, and the log-rank test was conducted to examine the significance of the difference between survival curves. Univariate and multivariate analyses were performed to determine the prognostic value between different clinicopathological factors and survival. Statistical significance was set at $P<0.05$.

\section{Results}

\section{Aberrant LIMCHI down-regulation in TCGA and the clinical LUAD cohort}

We first determined the LIMCH1 mRNA expression level using TCGA data. As shown in Figure 2A-B, among 57 paired LUAD samples, decreased LIMHC1 expression was observed frequently in tumor tissues than in the adjacent non-tumorous tissues, the LIMHC1 level was consistently down-regulated in 524 samples of LUAD 
tissues compared with the 59 samples of normal lung tissues. We also observed a lower LIMCH1 level in a total of 7 GEO datasets from the Oncomine database (Table 1).

Table 1. The relative expression of $\mathrm{LIMCHI}$ between lung adenocarcinoma tissues and adjacent normal tissues in Oncomine database

\begin{tabular}{lllll}
\hline GEO datasets & Normal & Tumor & $p$ value & Fold change \\
\hline GSE3398 & 6 & 39 & $5.05 \mathrm{E}-08$ & -4.318 \\
GSE7670 & 30 & 27 & $3.02 \mathrm{E}-13$ & -3.791 \\
GSE10072 & 49 & 58 & $3.33 \mathrm{E}-25$ & -3.336 \\
GSE2514 & 19 & 20 & $7.88 \mathrm{E}-08$ & -3.363 \\
GSE19188 & 65 & 45 & $5.20 \mathrm{E}-16$ & -4.087 \\
GSE32863 & 58 & 58 & $1.94 \mathrm{E}-22$ & -2.968 \\
GSE31210 & 20 & 226 & $1.01 \mathrm{E}-12$ & -2.177 \\
\hline
\end{tabular}

We performed IHC to test the protein levels of LIMCH1 in LUAD tissues. As shown in Figure 3, LIMCH1 was positively stained in the cytoplasmic, membranous, and perinuclear regions in the LUAD samples. In 9 paired LUAD tissues (Figure 3C-D), we observed a higher percentage of expression in adjacent normal tissues (7/9) than in the matched tumor tissues (2/9). Moreover, IHC evidence from the Human Protein Atlas database supported the downregulation of LIMCH1 protein in LUAD tissues (Figure 3A-B).

\section{Diagnostic and Prognostic Roles of LIMCHI}

In the TCGA cohort, ROC curves showed that
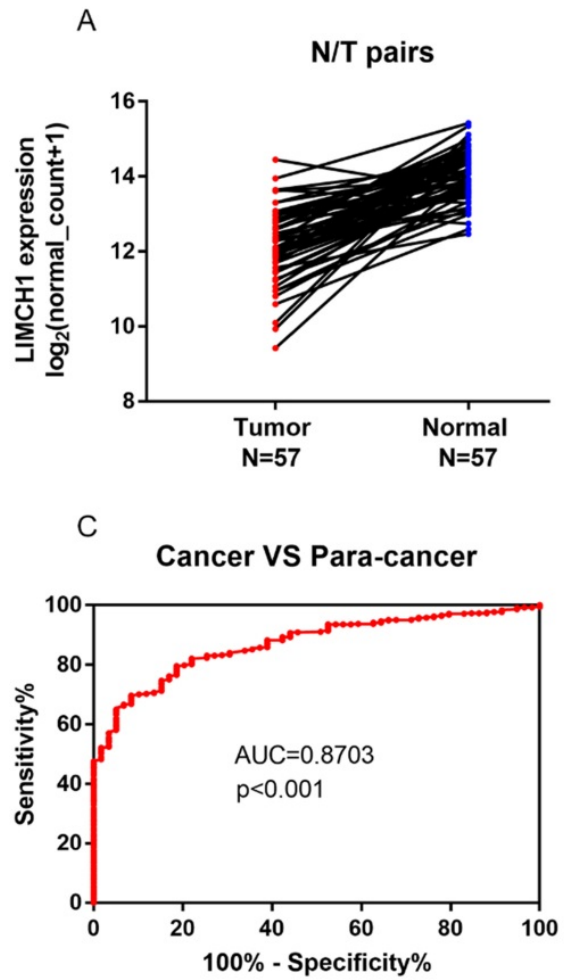

LIMCH1 expression was a powerful diagnostic indicator (Figure 2C, AUC $=0.870, P<0.001$ ). Survival analysis also showed that low LIMCH1 expression was associated with poor prognosis in patients with LUAD (Figure 2D). Multivariate Cox regression analysis (Table 2) showed that the T stage (HR 2.009, 95\% confidence interval [CI] 1.364-2.958), N stage (HR 2.313, 95\% CI 1.715-3.119), and LIMCH1 expression (HR 0.689, 95\% CI 0.512-0.927) were significant independent prognostic predictors $(P<0.05)$ for the LUAD cohort from TCGA.

\section{LIMCHI Expression and Clinicopathological Factors}

In our clinical cohort, the patients' age at surgery ranged from 35 to 87 years, with a median age of 61 years. Ninety-five patients $(48.5 \%)$ were female, and 70 patients $(35.7 \%)$ had a smoking history. One hundred twenty-five patients $(63.8 \%)$ died during a median follow-up of 48 months (3-104 months). Patients were divided into 2 groups (low expression vs high expression) based on the IHC staining data. Lower LIMCH1 expression positively correlated with several parameters, including pleural invasion, tumor length, tumor differentiation grade, clinical tumor stage and therapeutic strategy (all $P<0.05$ ). However, no significant association was observed in age, gender, or smoking history between these 2 groups (Table 3).
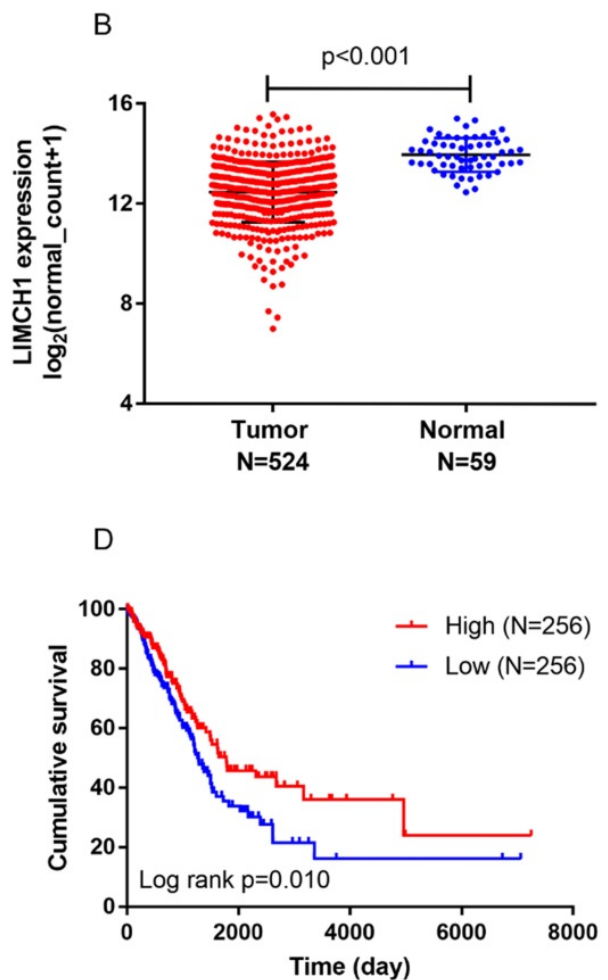

Figure 2. Aberrant LIMCHI expression, prognostic value, and survival analysis in the TCGA-LUAD cohort. (A) Low expression of LIMCH1 in 57 paired of LUAD tissues. (B) LIMCHI mRNA expression decreased in LUAD tissues than normal lung tissues. Diagnostic value (C) and survival analysis (D) of patients from the TCGA cohort. 
A

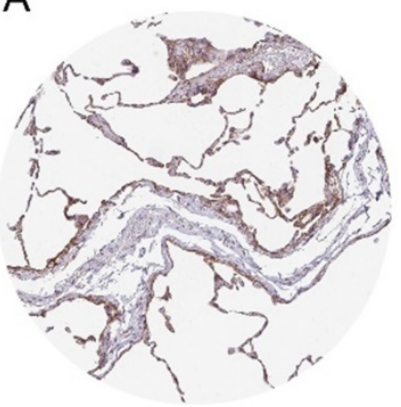

E

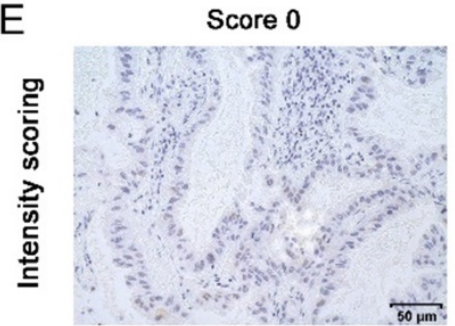

I

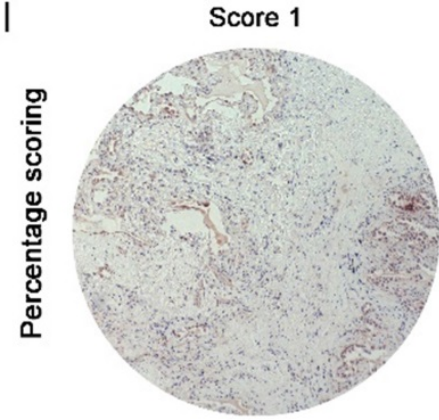

B
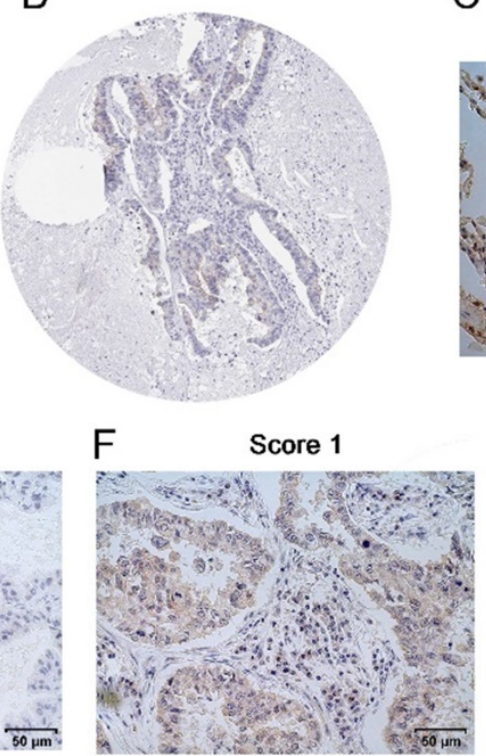

\section{$J$}

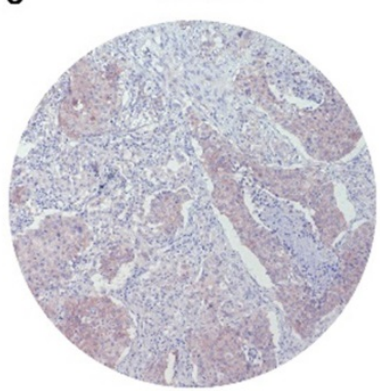

C
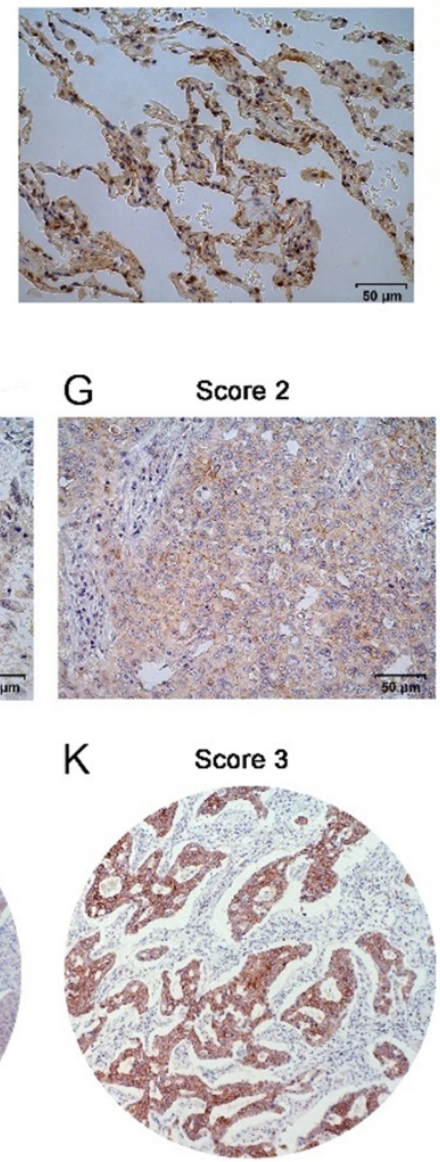

D

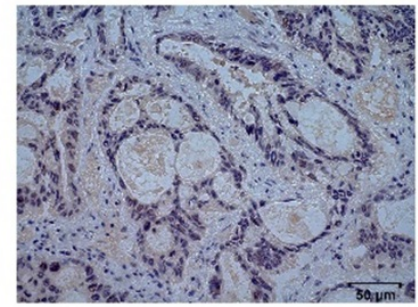

$\mathrm{H}$

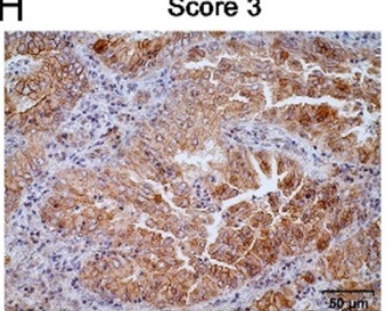

L

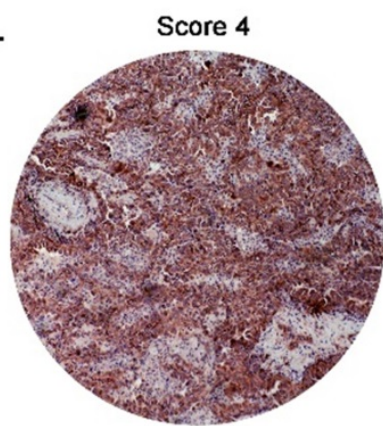

Figure 3. The protein expression of LIMCHI in LUAD tissues and non-tumor tissues. IHC staining of normal lung tissue (A) and LUAD tissue (B) from HPA datasets. Representative images of IHC staining of LIMCHI in paired adjacent normal tissues (C) and LUAD tissues (D) (Magnification $\times 400)$. Tumor tissues without LIMCHI staining which score $=0(\mathbf{E})$; weak staining of LIMCHI which score=1 $(\mathbf{F})$; moderate staining of LIMCHI which score $=2(\mathbf{G})$; strong staining of LIMCHI which score $=3(\mathbf{H})$; (Magnification $\times 400$ ). Representative images of step percentages of LIMCHI staining in $1 \%-25 \%$ which score=1 $(\mathrm{I})$; LIMCHI staining percentage of $26 \%-50 \%$ which score $=2(\mathrm{~J})$; LIMCHI staining percentage of $51 \%-75 \%$ which score $=3(\mathbf{K})$, LIMCHI staining percentage of $>75 \%$ which score $=4$ (L); (Magnification $\times 40)$. Bar scale: $50 \mu$ m.

Table 2. Multivariate Cox proportional hazards regression of overall survival in TCGA-LUAD cohort

\begin{tabular}{lccc}
\hline Variables & HR & $95 \%$ CI & $p$ value \\
\hline $\mathbf{T}$ & 2.009 & $1.364-2.958$ & $<0.001$ \\
T1-T2 vs T3-T4 & & & \\
$\mathbf{N}$ & 2.313 & $1.715-3.119$ & $<0.001$ \\
N0 vs N1-3 & & & \\
$\mathbf{M}$ & 1.681 & $0.956-2.953$ & 0.071 \\
M0 vs M1 & 0.689 & $0.512-0.927$ & 0.014 \\
LIMCH1 expression & & & \\
\hline
\end{tabular}

\section{Survival Analysis}

The survival curves (Figure 4) demonstrated that the patients with higher LIMCH1 expression had better prognosis $(P=0.022)$. We conducted univariate and multivariate analyses to examine the independent prognostic significance of LIMCH1 expression. The results of univariate analysis indicated that pleural invasion, differentiation grade, tumor length, T stage, $\mathrm{N}$ stage, TNM stage, and LIMCH1 expression were associated with overall survival in patients with
LUAD, and multivariate analysis confirmed that LIMCH1 expression (HR 0.545, 95\% CI 0.358-0.830), N stage (HR 3.618, 95\% CI 1.281-10.217), and TNM stage (HR 3.662, 95\% CI 2.261-5.931) were independent prognostic factors (Table 4 ).

Table 3. Correlations between LIMCHI expression and clinical parameters of 196 patients with lung adenocarcinoma

\begin{tabular}{lllll}
\hline Category & Case $(\mathrm{N}, \%)$ & \multicolumn{2}{l}{ LIMCH1 expression } & $p$ \\
\cline { 2 - 4 } & $196(100 \%)$ & $\begin{array}{l}\text { Low } \\
(\mathrm{n}=111)\end{array}$ & $\begin{array}{l}\text { High } \\
(\mathrm{n}=85)\end{array}$ & value \\
\hline $\begin{array}{l}\text { Age (years) } \\
\leq 60\end{array}$ & $94(48.0)$ & 52 & 42 & 0.722 \\
$>60$ & $102(52.0)$ & 59 & 43 & \\
Gender & $95(48.5)$ & 56 & 39 & 0.526 \\
Male & $101(51.5)$ & 55 & 46 & \\
$\begin{array}{l}\text { Female } \\
\text { Smoking }\end{array}$ & $126(64.3)$ & 70 & 56 & 0.683 \\
Never & $70(35.7)$ & 41 & 29 & \\
$\begin{array}{l}\text { Ever } \\
\text { Pleural invasion }\end{array}$ & $141(71.9)$ & 71 & 70 & 0.005 \\
No & $55(28.1)$ & 40 & 15 & \\
Yes & & & & 0.046 \\
Tumor length (cm) & $97(49.5)$ & 47 & 42 & \\
$\leq 4$ & & & & \\
\hline
\end{tabular}




\begin{tabular}{lllll}
\hline Category & Case (N, \%) & \multicolumn{2}{l}{ LIMCH1 expression } & $p$ \\
\cline { 2 - 4 } & $196(100 \%)$ & $\begin{array}{l}\text { Low } \\
(\mathrm{n}=111)\end{array}$ & $\begin{array}{l}\text { High } \\
(\mathrm{n}=85)\end{array}$ & \\
\hline$>4$ & $99(50.5)$ & 64 & 43 & 0.677 \\
$\begin{array}{l}\text { Location } \\
\text { Central }\end{array}$ & $49(25.0)$ & 29 & 20 & \\
$\begin{array}{l}\text { Peripheral } \\
\text { Differentiation }\end{array}$ & $147(75.0)$ & 82 & 65 & \\
Well or moderate & $51(26.0)$ & 22 & 29 & 0.024 \\
$\begin{array}{l}\text { Poor } \\
\text { T stage }\end{array}$ & $145(74.0)$ & 89 & 56 & \\
T1-T2 & & & & 0.273 \\
T3-T4 & $146(74.5)$ & 86 & 60 & \\
N stage & $50(25.5)$ & 25 & 25 & \\
N0 & & & & 0.004 \\
N1-N3 & $26(13.3)$ & 8 & 18 & \\
TNM stage & $170(86.7)$ & 103 & 67 & \\
I/II & & & & 0.001 \\
III & $97(49.5)$ & 66 & 31 & \\
Treatment & $99(50.5)$ & 45 & 54 & \\
Surgery only & & & & \\
Surgery with chemotherapy & $25(12.8)$ & 7 & 18 & 0.000 \\
Surgery with chemoradiotherapy & $102(52.0)$ & 71 & 31 & \\
\hline
\end{tabular}

\section{Bioinformatics Analyses}

We selected 312 co-expression genes contained in both the Affymetrix GeneChip Human Genome U133 Plus 2.0 (containing 1794 datasets) by MEM and the cBioPortal TCGA-LUAD dataset (provisional) for the bioinformatics analyses (Figure 5A). Functional annotation (GO and KEGG pathway) was then carried out using DAVID. The top 20 significant enrichment terms are shown in Figure 5C-D. These co-expressed genes were enriched in 'actin cytoskeleton organization', 'positive regulation of apoptotic process' and 'negative regulation of cell proliferation' in the biological processes; in the 'cell-cell junction', 'cytoplasm', and 'focal adhesion' in cellular components; in 'protein kinase binding' , 'actin binding', and 'GTPase activity' in the molecular functions. KEGG pathway analysis revealed enriched terms as 'proteoglycans in cancer', 'signaling pathways regulating pluripotency of stem cells', and 'Wnt signaling pathway' (Figure 5B), suggesting that those genes were involved in development of LUAD.

To identify the mechanisms underlying LIMCH1 expression, we also conducted GSEA. Eight hallmarks were significantly enriched in the low LIMCH1 expression group, including mTORC1 signaling, MYC targets, DNA repair, and G2M checkpoint (Figure 6A-E and Table 5). These analyses indicated that LIMCH1 was closely associated with cell cycle and proliferation to affect LUAD.

\section{Discussion}

LUAD is a highly malignant disease with various genetic backgrounds. Despite recent advances in precision medicine, the 5-year survival rate remains low. Therefore, novel prognosis biomarkers are urgently needed to improve the outcome of patients with LUAD. In this research, we demonstrated that LIMCH1 was downregulated in mRNA and protein levels in LUAD tissues. The low percentage of LIMCH1 staining was an independent risk factor for poor outcome in patients with LUAD. We also explored the possible mechanism for this finding.

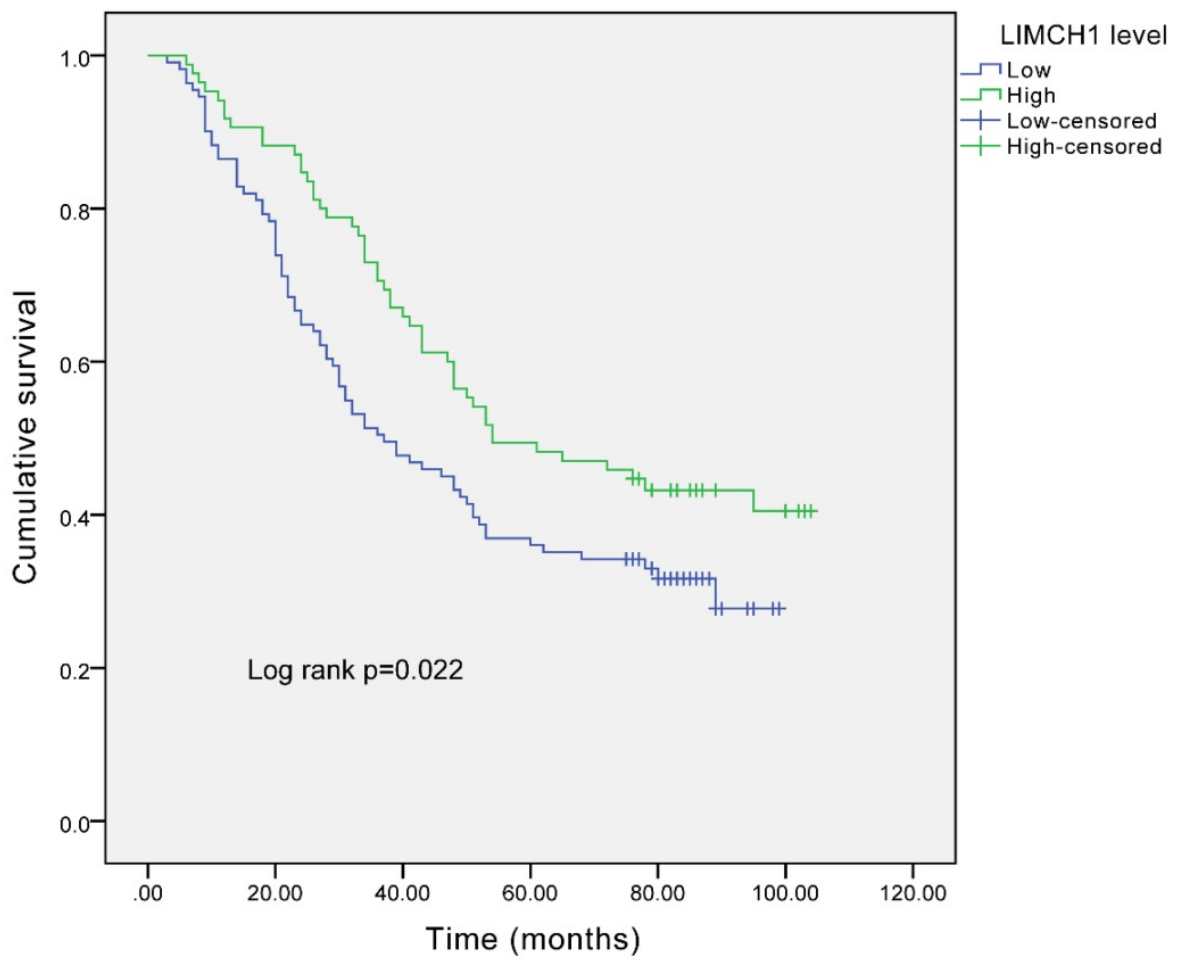

Figure 4. Survival analysis of LUAD patients with different expressions of LIMCHI based on IHC staining. 
Table 4. Univariate and multivariate analyses of the prognostic factors in 196 patients with lung adenocarcinoma

\begin{tabular}{|c|c|c|c|c|c|c|}
\hline & \multicolumn{3}{|c|}{ Univariate analysis } & \multicolumn{3}{|c|}{ Multivariate analysis } \\
\hline & $p$ value & HR & $95 \% \mathrm{CI}$ & $p$ value & HR & $95 \% \mathrm{CI}$ \\
\hline Age ( $\leq 60$ years vs $>60$ years) & 0.177 & 1.276 & $0.895-1.818$ & & & \\
\hline Gender (female vs male) & 0.958 & 0.991 & $0.697-1.408$ & & & \\
\hline Smoking (ever vs never) & 0.524 & 1.126 & $0.781-1.624$ & & & \\
\hline Pleural invasion (yes vs no) & $<0.001$ & 2.024 & $1.398-2.930$ & 0.297 & 1.235 & $0.831-1.836$ \\
\hline Differentiation (well/moderate vs poor) & $<0.001$ & 2.586 & $1.614-4.141$ & 0.248 & 1.345 & $0.814-2.222$ \\
\hline Tumor length $(\leq 4 \mathrm{~cm}$ vs $>4 \mathrm{~cm})$ & $<0.001$ & 2.051 & $1.428-2.947$ & 0.508 & 1.167 & $0.739-1.842$ \\
\hline Location (central vs peripheral) & 0.264 & 0.799 & 0.538-1.185 & & & \\
\hline T stage (T1/T2 vs T3/T4) & 0.005 & 1.725 & $1.176-2.531$ & 0.063 & 0.617 & $0.371-1.026$ \\
\hline N stage (N0 vs N1/N2/N3) & $<0.001$ & 8.172 & $3.010-22.185$ & 0.015 & 3.618 & $1.281-10.217$ \\
\hline TNM stage (I/II vs III) & $<0.001$ & 3.250 & $2.234-4.727$ & $<0.001$ & 3.662 & $2.261-5.931$ \\
\hline LIMCH1 (High vs Low) & 0.024 & 0.660 & $0.460-0.947$ & 0.005 & 0.545 & $0.358-0.830$ \\
\hline
\end{tabular}

A

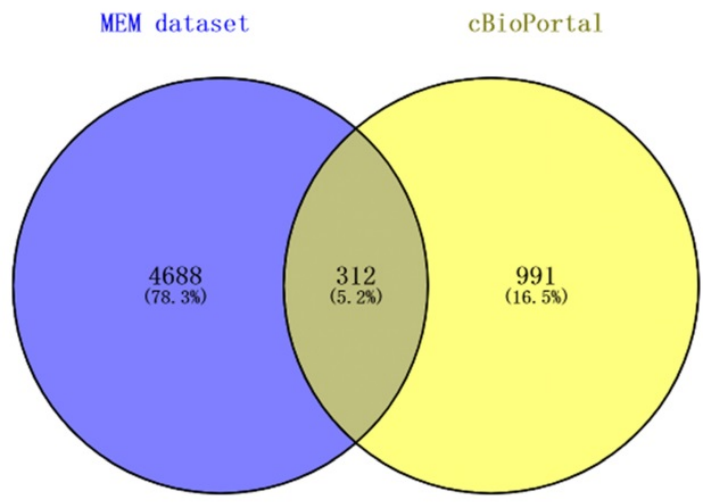

C

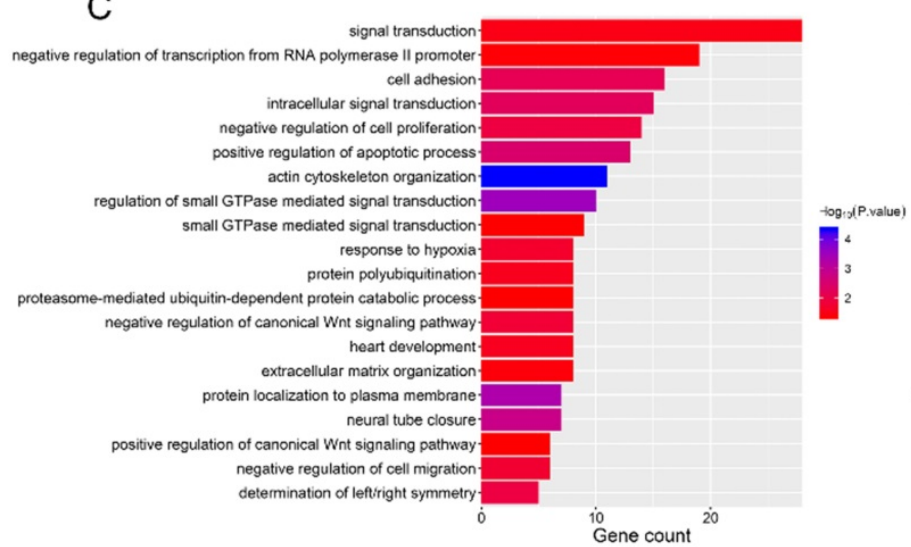

B
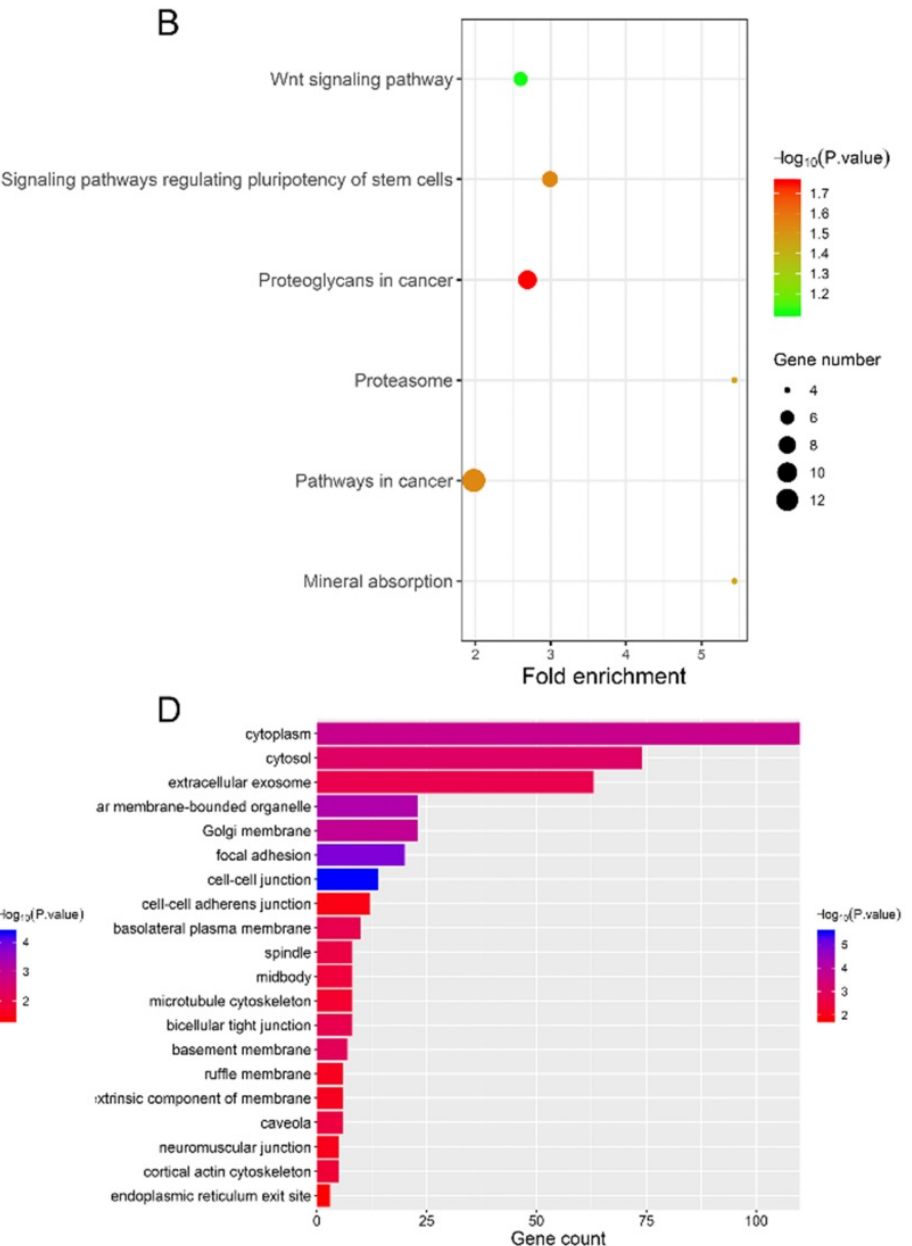

Figure 5. DAVID analysis of co-expressed genes. (A) the intersection of co-expressed genes between MEM and cBioPortal datasets. (B) KEGG analysis revealed significantly enriched pathways. GO terms of Biological Process (C) and Cellular Component (D).

Table 5. Gene Set Enrichment Analysis of LIMCHI in lung adenocarcinoma

\begin{tabular}{|c|c|c|c|c|}
\hline NAME & ES & NES & $\begin{array}{l}\text { NOM } \\
\text { p-value }\end{array}$ & $\begin{array}{l}\text { FDR } \\
\text { q-value }\end{array}$ \\
\hline HALLMARK_MTORC1_SIGNALING & 0.530 & 1.942 & 0.002 & 0.0813 \\
\hline HALLMARK_MYC_TARGETS_V1 & 0.601 & 1.914 & 0.022 & 0.052 \\
\hline HALLMARK_MYC_TARGETS_V2 & 0.711 & 1.893 & 0.006 & 0.042 \\
\hline HALLMARK_DNA_REPAIR & 0.487 & 1.881 & 0.004 & 0.035 \\
\hline HALLMARK_E2F_TARGETS & 0.710 & 1.835 & 0.012 & 0.0472 \\
\hline HALLMARK_G2M_CHECKPOINT & 0.619 & 1.701 & 0.044 & 0.118 \\
\hline HALLMARK_GLYCOLYSIS & 0.381 & 1.537 & 0.038 & 0.192 \\
\hline HALLMARK_UV_RESPONSE_UP & 0.331 & 1.459 & 0.049 & 0.220 \\
\hline
\end{tabular}

LIMCH1, which also named KIAA1102, encodes actin stress-fibers associated protein. It promotes the phosphorylation of regulatory subunit MRLC/MYL9 to activate cell migration. Several recent studies reported that LIMCH1 expression was associated with breast cancer, clear cell renal cell carcinoma, and lung cancer. However, its clinical prognostic value in patients with LUAD remains unclear. 

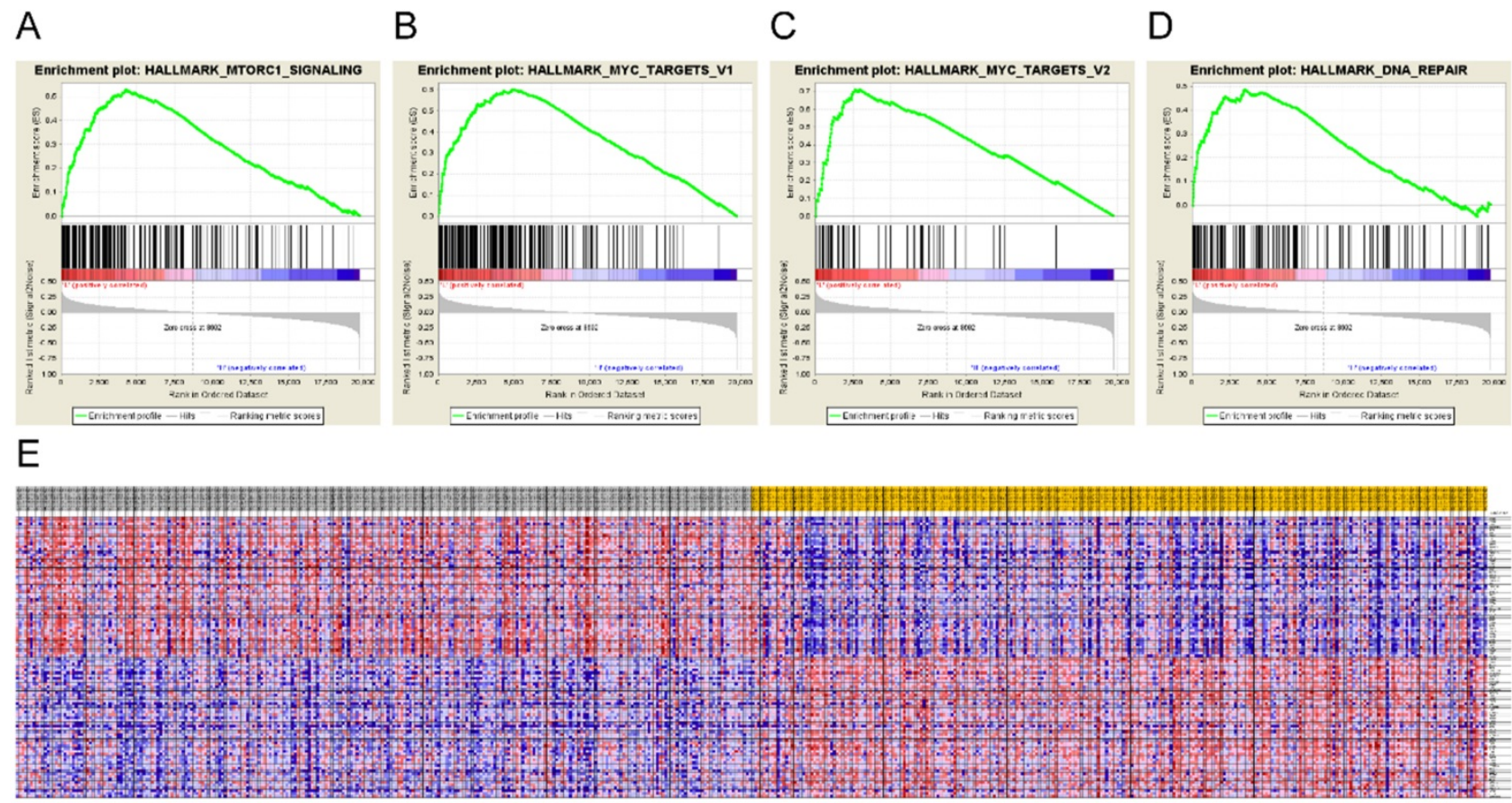

Figure 6. Gene Set Enrichment Analysis of LIMCHI. (A-D) The most significantly associated pathways. (A) MTORC1 signaling; (B) MYC targets; (C) DNA repair; (D) G2M checkpoint; (E) Heat Map of the top 100 genes.

In this study, we first showed that LIMCH1 expression is downregulated in LUAD tissues by performing IHC staining in our cohort. Data mining from TCGA, Oncomine, and HPA datasets also supports our results. To our knowledge, this is the first study to discuss the protein expression level of LIMCH1 in LUAD. We found that LIMCH1 plays a role in tumorigenesis and tumor progression. Based on the strong evidence of differentiative expressions in tissues and of the actin stress binding function in specific cell types, we explored the prognostic role in patients with LUAD. Our results show that reduced LIMCH1 expression is associated with pleural invasion, more substantial tumor length, lower differentiation grade, and more advanced tumor stage. In both TCGA-LUAD and our LUAD datasets, patients with lower LIMCH1 expression have shorter survival times. Cox regression analysis revealed that LIMCH1 expression independently predicts poor overall survival.

A previous study of the molecular mechanisms involved in cancer development, using yeast 2-hybrid screen found that LRIG proteins interacted with LIMCH1 peptides [11]. Zhang et al. reported that LIMCH1 was reciprocally co-immunoprecipitated with HUWE1 to ubiquitinated p53. Degradation of p53 affects downstream proteins to regulate cell proliferation [10]. In our study, GO and KEGG analyses of co-expressed genes were conducted to identify the function of LIMCH1. Gene-annotation enrichment analysis showed that LIMCH1 co-expressed gene function is associated with cell adhesion and actin cytoskeleton organization, which is consistent with previous studies. KEGG analysis demonstrated that these genes are involved in several cancer-related pathways, and GSEA identified 8 hallmark pathways, including MTORC1, MYC targets, DNA repair, G2M checkpoint signaling. DNA repair and cell cycle seriously affected cell proliferation, which was associated with LUAD progression. Further studies are needed to verify these mechanisms in vitro and in vivo.

There are still certain limitations in this current study. First, the clinical LUAD cohort was obtained from our single center, and the clinical parameters were collected retrospectively. Thus, the selection bias could not be avoided. More prospective multi-center studies are needed to validate these results. Second, we selected just one core per tumor to construct the tissue microarray, thus, the tumor heterogeneity could be a challenge. Future studies should add more cores from each tumor block to eliminate the problem to a large part.

In conclusion, our current study demonstrated that LIMCH1 expression is associated with aggressive tumorigenesis, and that decreased expression of LIMCH1 predicts a shorter overall survival time, which is an independent prognostic factor in patients with LUAD. LIMCH1 appears to be a novel prognostic target for LUAD, but more real-world studies are needed to confirm the critical mechanisms underlying cancer development in patients with LUAD. 


\section{Abbreviations}

LUAD: lung adenocarcinoma; LUSC: lung squamous carcinoma; IHC: immunohistochemistry; NSCLC: non-small lung cancer; TCGA: The Cancer Genome Atlas; GEO: Gene Expression Omnibus; GSEA: gene set enrichment analysis; FDR: false discovery rate; TKIs: tyrosine kinase inhibitor; HR: hazard ratio; 95\% CI: 95\% confidence interval; ROC: receiver operating characteristic; AUC: area under the curve; Espin: ESPN; ESCC: esophageal squamous cell carcinoma; TWF1: Twinfilin-1; NM-II: non-muscle myosin II; WHO: World Health Organization's; TMA: tissue microarray; DAB: diaminobenzidine; IRS: immunoreactive scoring; TNM stage: tumor-nodemetastasis stage.

\section{Acknowledgments}

This work was supported by grants from the National Natural Science Foundation of China (81972179) and the Zhejiang Provincial Science and Technology Program (2019C03042).

\section{Competing Interests}

The authors have declared that no competing interest exists.

\section{References}

1. Chen W, Zheng R, Baade PD, et al. Cancer statistics in China, 2015. CA Cancer J Clin. 2016; 66: 115-32.

2. Siegel RL, Miller KD, Jemal A. Cancer statistics, 2020. CA Cancer J Clin. 2020; 70: 7-30.

3. Bray F, Ferlay J, Soerjomataram I, et al. Global cancer statistics 2018: GLOBOCAN estimates of incidence and mortality worldwide for 36 cancers in 185 countries. CA Cancer J Clin. 2018; 68: 394-424.

4. Li SH, Lu HI, Huang WT, et al. An actin-binding protein ESPN is an independent prognosticator and regulates cell growth for esophageal squamous cell carcinoma. Cancer Cell Int. 2018; 18: 219.

5. Kaishang Z, Xue P, Shaozhong Z, et al. Elevated expression of Twinfilin-1 is correlated with inferior prognosis of lung adenocarcinoma. Life Sci. 2018; 215: $159-69$.

6. Lin $\mathrm{YH}$, Zhen $\mathrm{YY}$, Chien KY, et al. LIMCH1 regulates nonmuscle myosin-II activity and suppresses cell migration. Mol Biol Cell. 2017; 28: 1054-65.

7. Bersini S, Lytle NK, Schulte R, et al. Nup93 regulates breast tumor growth by modulating cell proliferation and actin cytoskeleton remodeling. Life sci. alliance. 2020; 3: e201900623.

8. Eckel-Passow JE, Serie DJ, Bot BM, et al. ANKS1B is a smoking-related molecular alteration in clear cell renal cell carcinoma. BMC Urol. 2014; 14: 14

9. Liu C, Zhang $\mathrm{YH}$, Huang $\mathrm{T}$, et al. Identification of transcription factors that may reprogram lung adenocarcinoma. Artif Intell Med. 2017; 83: 52-7.

10. Zhang $\mathrm{Y}$, Zhang $\mathrm{Y}, \mathrm{Xu} \mathrm{H}$. LIMCH1 suppress the growth of lung cancer by interacting with HUWE1 to sustain p53 stability. Gene. 2019; 712: 143963.

11. Karlsson T, Kvarnbrink S, Holmlund C, et al. LMO7 and LIMCH1 interact with LRIG proteins in lung cancer, with prognostic implications for early-stage disease. Lung cancer. 2018; 125: 174-84.

12. Travis WD, Brambilla E, Burke AP, et al. Introduction to The 2015 World Health Organization Classification of Tumors of the Lung, Pleura, Thymus, and Heart. J Thorac Oncol. 2015; 10: 1240-2.

13. Sun R, Ma C, Wang W, et al. Upregulation of desmoglein 2 and its clinical value in lung adenocarcinoma: a comprehensive analysis by multiple bioinformatics methods. PeerJ. 2020; 8: e8420.

14. Goldman M, Craft B, Brooks A, et al. The UCSC Xena Platform for cancer genomics data visualization and interpretation. BioRxiv 2018; [Epub ahead of print].

15. Rhodes DR, Yu J, Shanker K, et al. ONCOMINE: a cancer microarray database and integrated data-mining platform. Neoplasia. 2004; 6: 1 .

16. Uhlén M, Fagerberg L, Hallström BM, et al. Tissue-based map of the human proteome. Science. 2015; 347: 1260419 .

17. Uhlen $\mathrm{M}$, Zhang $\mathrm{C}$, Lee $\mathrm{S}$, et al. A pathology atlas of the human cancer transcriptome. Science. 2017; 357: eaan2507.
18. Adler P, Kolde R, Kull M, et al. Mining for coexpression across hundreds of datasets using novel rank aggregation and visualization methods. Genome Biol. 2009; 10: R139.

19. Gao J, Aksoy BA, Dogrusoz U, et al. Integrative analysis of complex cancer genomics and clinical profiles using the cBioPortal. Sci Signal. 2013; 6: pl1.

20. Oliveros JC. VENNY. An interactive tool for comparing lists with Venn Diagrams. http://bioinfogp.cnb.csic.es/tools/venny/index.html. 2007.

21. Sherman BT, Lempicki RA. Systematic and integrative analysis of large gene lists using DAVID bioinformatics resources. Nat Protoc. 2009; 4: 44. 